\title{
Dynamics of the corotating vortices in dipolar Bose-Einstein condensates in the presence of dissipation
}

\author{
S. Gautam ${ }^{1}$ \\ ${ }^{1}$ Instituto de Física Teórica, UNESP - Universidade Estadual Paulista, \\ 01.140-070 São Paulo, São Paulo, Brazil
}

(Dated: April 25, 2022)

\begin{abstract}
We study the dynamics of a single and a corotating vortex pair in a dipolar Bose-Einstein condensate in the framework of dissipative Gross-Pitaevskii equation. This simple model enables us to simulate the effect of finite temperature on the vortex dynamics. We study the effect of dipolar interactions on the dynamics of a single vortex in the presence of phenomenological dissipation. In the case of a corotating vortex pair, an initial asymmetry in the locations of the vortices can lead to different decay rates for the constituent vortices as is the case for the condensates interacting via pure contact interactions. We observe that the anisotropic interaction between the component vortices manifests itself as the perceptible difference in the trajectories traversed by the vortices in the condensate at finite temperatures.

PACS numbers: 03.75.Kk, 05.30.Jp, 67.85.De
\end{abstract}

\section{INTRODUCTION}

Quantized vortices in the Bose-Einstein condensates (BECs), which unequivocally prove the superfluid nature of these systems, can be produced experimentally using a variety of methods [1 5 . Besides a single vortex [1, 2] and a vortex lattice consisting of corotating vortices [4, creation of the vortex-antivortex pairs or vortex dipoles has also been observed during (a) the motion of the condensate across the Gaussian obstacle potential [6], (b) the decay of the dark soliton in the quasi twodimensional condensates [7, and (c) the rapid quench of the condensate through the condensation temperature [8, 9]. Freilich et al. have performed the experimental study of the real time dynamics of a single vortex as well as a vortex dipole 9]. In a recent experiment, the dynamics of 2-4 corotating vortices was also studied [10]. At zero temperature, the dynamics of vortex dipoles has been investigated theoretically in Refs. 11113. The generation and energetic stability of the vortex dipoles in phase-separated binary condensates was also theoretically investigated in a couple of our earlier works 14. At finite temperature, an off-centered vortex in a non-rotating condensate moves out of the condensate following a spiral trajectory, and it has been confirmed by various theoretical investigations [15]22]. Recently we studied the dynamics of a single vortex as well a vortex pair in the BECs at finite temperature using the stochastic Gross-Pitaevskii equation (SGPE) [23].

All the studies mentioned in the previous paragraph have dealt with the BECs interacting via short range and isotropic contact interactions. Dipolar interactions were not considered in any of these studies. The primary motivation of the present work is to study the dynamics of a single and a pair of corotating vortices in the dipolar Bose-Einstein condensates (DBECs) at finite temperatures. A lot of experimental and theoretical studies have been done on the dipolar quantum gases since the first experimental realization of the Bose-Einstein condensation in a gas of chromium $\left({ }^{52} \mathrm{Cr}\right)$ atoms, which have permanent magnetic dipole moments 24 26. In addition to ${ }^{52} \mathrm{Cr}$, Bose-Einstein condensation in the systems with stronger dipolar interactions like dysprosium $\left({ }^{164} \mathrm{Dy}\right)[27$ and erbium $\left({ }^{168} \mathrm{~Eb}\right)[28$ has also been observed experimentally. The various developments in the field of the dipolar quantum degenerate gases have been reviewed in Refs. [29, 30]. Dipolar interaction is characterized by its long range and anisotropic nature, which is not the case with contact interactions. Also, the inter-particle interaction in dipolar condensates is momentum dependent as all the partial waves contribute to the scattering amplitude 29. This leads to the emergence of roton like excitations in the system [31 34]. The presence of roton like excitations is responsible for variety of effects like lowering of the Landau critical speed 35. below the speed of sound for sufficiently large particle number [33], density fluctuations at defects like vortices 32, 36, roton instability [31, 34, 37, 38, enhancement of the density fluctuations in two-dimensional DBECs [39, and anisotropic superfluidity [40, 41] which in turn leads to the anisotropic merging and splitting in quasi two-dimensional DBECs [42. At zero temperature, the effect of dipolar interactions and the relative strength of dipolar and contact interactions on the structure and stability of the vortices in the DBECs has been theoretically investigated [32, 36, 43]. The dynamical instability of the rotating dipolar condensates, which can lead to vortex lattice formation, has been studied in Ref. [4]. The vortex lattice formation in the rotating quasi two dimensional DBECs has also been studied theoretically [45, 46].

In the present work, we study the dynamics of a single and a corotating vortex pair in the framework of dissipative Gross-Pitaevskii equation (DGPE). The DGPE is the simplest model that can simulate the effect of the finite temperature on the vortex dynamics in DBECs [47. The microscopic origin of the dissipation in the non-dipolar condensates at finite temperature conden- 
sates has been discussed in Refs. [48, 49].

The paper is organized as follows- We provide the mean-field description of DBECs in Sec. III in the presence of phenomenological dissipation. Here we discuss the quasi two-dimensional non-local dissipative GrossPitaevskii equation (DGPE) which we employ to study the dipolar Bose-Einstein condensate (DBEC) with an arbitrary direction of polarization. In Sec. III. we study the dynamics of a single and a corotating vortex pair in the presence of dissipation. We conclude by providing a summary of results in Sec. IV.

\section{MEAN FIELD DESCRIPTION OF DIPOLAR BOSE-EINSTEIN CONDENSATE}

In the mean field regime, a dipolar Bose-Einstein condensate (DBEC) at finite temperature can be modeled by the non-local DGPE [16, 29, 30.

$$
\begin{aligned}
i \hbar \frac{\partial \Psi(\mathbf{x}, t)}{\partial t}= & (1-i \gamma)\left[-\frac{\hbar^{2} \nabla^{2}}{2 m}+V(\mathbf{x})+g|\Psi(\mathbf{x}, t)|^{2}+\right. \\
& \left.\int U_{\mathrm{dd}}\left(\mathbf{x}-\mathbf{x}^{\prime}\right)\left|\Psi\left(\mathbf{x}^{\prime}, t\right)\right|^{2} d \mathbf{x}^{\prime}\right] \Psi(\mathbf{x}, t), \quad(1)
\end{aligned}
$$

where $\Psi(\mathbf{x}, t)$ is the wave function of the condensate and $\gamma$ is the dissipation introduced by the thermal cloud. The last term in the square brackets accounts for the long range dipole-dipole interaction. When all the dipoles point in the same direction, i.e., the dipolar gas is polarized, the dipole-dipole interaction strength is

$$
U_{\mathrm{dd}}=\frac{C_{\mathrm{dd}}}{4 \pi} \frac{1-3 \cos ^{2} \theta}{\left|\mathbf{x}-\mathbf{x}^{\prime}\right|^{3}}
$$

where $\theta$ is the angle between the direction of polarization and relative position vector of the dipoles. The coupling constant $C_{\mathrm{dd}}=12 \pi \hbar^{2} a_{\mathrm{dd}} / m$, where $a_{\mathrm{dd}}$ is the length characterizing the strength of the dipolar interactions and $m$ is the mass of the atom. For the dipolar gas consisting of atoms with permanent magnetic dipole moment $\chi$ like ${ }^{52} \mathrm{Cr}, a_{\mathrm{dd}}=\mu_{0} \chi^{2} m /\left(12 \pi \hbar^{2}\right)$, where $\mu_{0}$ is the permeability of the free space. In the present work, we consider the DBEC trapped in a harmonic trapping potential $V(\mathbf{x})=m\left(\omega_{x}^{2} x^{2}+\omega_{y}^{2} y^{2}+\omega_{z}^{2} z^{2}\right) / 2$, where $\omega_{j}$ 's with $j=x, y, z$ are trapping frequencies along the three coordinate axes. The interaction strength $g=4 \pi \hbar^{2} a / m$, where $a$ is the $s$-wave scattering length, characterizes the contact interaction between atoms. The total number of atoms $N$ and energy $E$ are not conserved by Eq. (1) due to the presence of dissipation. For the sake of solving Eq. (1) numerically, we transform the DGPE into dimensionless form using following transformations:

$$
\mathbf{x}=\tilde{\mathbf{x}} a_{\mathrm{osc}}, t=\frac{2 \tilde{t} \omega^{-1}}{1+\gamma^{2}}, \Psi=\sqrt{N} \frac{\tilde{\Psi}}{a_{\mathrm{osc}}^{3 / 2}},
$$

where $a_{\mathrm{osc}}=\sqrt{\hbar /(m \omega)}$ with $\omega=\min \left\{\omega_{\mathrm{x}}, \omega_{\mathrm{y}}, \omega_{\mathrm{z}}\right\}$ is the oscillator length. The dimensionless DGPE for the DBEC is now of the form

$$
\begin{aligned}
& (i-\gamma) \frac{\partial \tilde{\Psi}}{\partial \tilde{t}}=\left[-\tilde{\nabla}^{2}+2 \tilde{V}+2 \tilde{g}|\tilde{\Psi}|^{2}+\right. \\
& \left.2 \int \tilde{U}_{\mathrm{dd}}\left(\tilde{\mathbf{x}}-\tilde{\mathbf{x}^{\prime}}\right)\left|\tilde{\Psi}\left(\tilde{\mathbf{x}^{\prime}}\right)\right|^{2} d \tilde{\mathbf{x}^{\prime}}\right] \tilde{\Psi},
\end{aligned}
$$

where $\tilde{V}=\left(\lambda_{x}^{2} \tilde{x}^{2}+\lambda_{y}^{2} \tilde{y}^{2}+\lambda_{z}^{2} \tilde{z}^{2}\right) / 2, \lambda_{j}=\omega_{j} / \omega$ with $j=x, y, z$ and $\tilde{g}=4 \pi \hbar a N /\left(m \omega a_{\text {osc }}^{3}\right)$. In the present work, we consider the DBEC in a quasi two-dimensional trap with $\lambda_{x}=\lambda_{y}=1 \ll \lambda_{z}$. In this case, the axial degrees of freedom of the system are frozen. We write $\tilde{\Psi}(\tilde{\mathbf{x}})=\tilde{\zeta}(z) \tilde{\psi}(\tilde{x}, \tilde{y})$ with $\tilde{\zeta}(\tilde{z})=\left(\lambda_{z} / \pi\right)^{1 / 4} e^{-\left(\lambda_{z} \tilde{z}^{2}\right) / 2}$ as the harmonic oscillator ground state along the axial direction. After integrating out the axial coordinate, we obtain the following two-dimensional equation [34, 40, 50, 51] in dimensionless form:

$$
\begin{aligned}
& (i-\gamma) \frac{\partial \tilde{\psi}}{\partial \tilde{t}}=\left\{-\tilde{\nabla}_{\tilde{r}}^{2}+2 \tilde{V}_{\tilde{r}}+2 \tilde{g}_{\tilde{r}}|\tilde{\psi}|^{2}+4 \sqrt{2 \pi \lambda_{z}} \tilde{a}_{\mathrm{dd}} N\right. \\
& \times \int \frac{d^{2} \tilde{k}_{\tilde{r}}}{4 \pi^{2}} e^{i \tilde{\mathbf{k}}_{\tilde{r}} \cdot \tilde{\mathbf{r}}} \tilde{n}\left(\tilde{\mathbf{k}}_{\tilde{r}}\right)\left[\cos ^{2}(\alpha) h_{2 d}^{\perp}\left(\frac{\tilde{\mathbf{k}}_{\tilde{r}}}{\sqrt{2 \lambda_{z}}}\right)\right. \\
& \left.\left.+\sin ^{2}(\alpha) h_{2 d}^{\|}\left(\frac{\tilde{\mathbf{k}}_{\tilde{r}}}{\sqrt{2 \lambda_{z}}}\right)\right]\right\} \tilde{\psi}
\end{aligned}
$$

where $\tilde{\nabla}_{\tilde{r}}^{2}=\partial^{2} / \partial \tilde{x}^{2}+\partial^{2} / \partial \tilde{y}^{2}, \quad \tilde{V}_{\tilde{r}}=\tilde{x}^{2} / 2+\tilde{y}^{2} / 2$, $\tilde{g}_{\tilde{r}}=\sqrt{\lambda_{z} / 2 \pi} \tilde{g}$, and $\tilde{a}_{\mathrm{dd}}=a_{\mathrm{dd}} / a_{\mathrm{osc}}$. Here we have considered an arbitrary direction of polarization in the $x z$ plane, which makes an angle $\alpha$ with the $z$ axis, and $h_{2 d}^{\perp}\left(\tilde{\mathbf{k}}_{\tilde{r}} / \sqrt{2 \lambda_{z}}\right)$ and $h_{2 d}^{\|}\left(\tilde{\mathbf{k}}_{\tilde{r}} / \sqrt{2 \lambda_{z}}\right)$ are defined as

$h_{2 d}^{\perp}\left(\frac{\tilde{\mathbf{k}}_{\tilde{r}}}{\sqrt{2 \lambda_{z}}}\right)=2-\frac{3 \sqrt{\pi} \tilde{k}_{\tilde{r}}}{\sqrt{2 \lambda_{z}}} \exp \left(\frac{\tilde{k}_{\tilde{r}}^{2}}{2 \lambda_{z}}\right) \operatorname{erfc}\left(\frac{\tilde{k}_{\tilde{r}}}{\sqrt{2 \lambda_{z}}}\right)$,
$h_{2 d}^{\|}\left(\frac{\tilde{\mathbf{k}}_{\tilde{r}}}{\sqrt{2 \lambda_{z}}}\right)=-1+\frac{3 \sqrt{\pi} \tilde{k}_{\tilde{\tilde{x}}}^{2}}{\sqrt{2 \lambda_{z}} \tilde{k}_{\tilde{r}}} \exp \left(\frac{\tilde{k}_{\tilde{r}}^{2}}{2 \lambda_{z}}\right) \operatorname{erfc}\left(\frac{\tilde{k}_{\tilde{r}}}{\sqrt{2 \lambda_{z}}}\right)$,

where $\tilde{k}_{\tilde{r}}=k_{r} a_{\text {osc }}$ and $\tilde{k}_{\tilde{x}}=k_{x} a_{\text {osc }}$. The scaled wavefunction is normalized to unity, i.e., $\int|\tilde{\psi}|^{2} d \tilde{x} d \tilde{y}=1$. For the sake of notational simplicity, from here on we will write the scaled variables without tildes unless stated otherwise. We use the time splitting Fourier spectral method to solve equation Eq. (5) [52]. The spatial and time step sizes employed in the present work are $0.11 a_{\text {osc }}$ and $0.0012 \omega^{-1}$ respectively. The wavefunction is normalized to unity after each iteration in time as it is no longer conserved in the presence of dissipation.

\section{DYNAMICS OF VORTICES}

Dynamics of a single vortex: We consider $1.5 \times 10^{5}$ atoms ${ }^{52} \mathrm{Cr}$ trapped in a harmonic trapping potential 
with $\omega_{x}=\omega_{y}=2 \pi \times 10 \mathrm{~Hz}$ and $\omega_{z}=2 \pi \times 100 \mathrm{~Hz}$. The back ground $s$-wave scattering length of ${ }^{52} \mathrm{Cr}$ is $100 a_{0}$, where as dipolar length is $16 a_{0}$ 29. In order to accentuate the effect of the dipolar interaction, we consider $a=50 a_{0}$ in the present work. In experiments, it can be achieved by tuning $s$-wave scattering length $a$ using magnetic Feshbach resonance [25]. The unit of length and time used in the present work are thus $a_{\text {osc }}=4.41 \mu \mathrm{m}$ and $\omega^{-1}=1.59 \times 10^{-2} \mathrm{~s}$ respectively. In order to generate the vortex in the system located at say $\left(x_{0}, y_{0}\right)$, we solve Eq. (5) in imaginary time $\tau=-i t$ in the absence of any dissipation. After each iteration in imaginary time, we imprint the phase corresponding to the presence of the vortex, i.e., we perform the transformation

$$
\psi(\tau+\delta \tau)=\exp \left(i \tan ^{-1} \frac{y-y_{0}}{x-x_{0}}\right)|\psi(\tau)|,
$$

where $\delta \tau$ is the increment in the imaginary time. The solutions obtained by this method for two different directions of polarization, $\alpha=0$ and $\alpha=\pi / 4$, are shown in the upper two rows of Fig. 1 In both cases, the vortex is imprinted at $\left(1.55 a_{\mathrm{osc}}, 0\right)$.
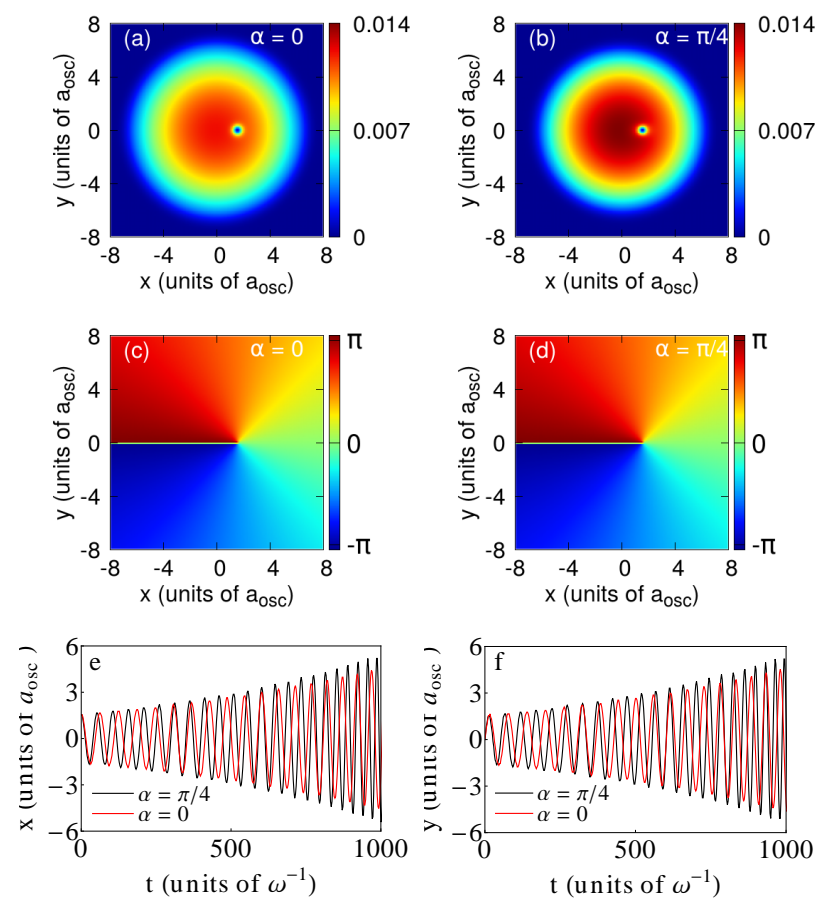

FIG. 1: The top row shows the density profiles of the condensate with a single vortex located at $\left(1.55 a_{\mathrm{osc}}, 0\right)$. The middle row shows the corresponding phase profiles. The angle $\alpha$, defining the direction of polarization, is equal to 0 for (a) and (c) and $\pi / 4$ for (b) and (d). The bottom row shows the dynamics of the vortex in the two cases. The red and black curves correspond to $\alpha=0$ and $\alpha=\pi / 4$ respectively.

It is evident from the Figs. 1(a) and (b) that for $\alpha=\pi / 4$ the peak density has increased, whereas the extent of the density profile has slightly decreased as compared to $\alpha=0$ case. This is due to the fact that for $\alpha=0$ the dipolar interactions are purely repulsive, whereas for $\alpha=\pi / 4$ the component of the dipoles along the $x$-axis interact attractively. Hence, the total dipolar interaction, which is still repulsive, is nevertheless weaker for $\alpha=\pi / 4$. This leads to the decrease in the condensate size and increase in the peak intensity. In order to study the dynamics of the vortex, we evolve these solutions in real time with non-zero dissipation. In the present work, we consider $\gamma=0.0023$ which is reasonable choice of the dissipation 22. We find that the vortex slowly spirals out of the condensate (see the bottom panel of Fig. 1) as is the case for the condensate interacting via pure contact interactions. This is not surprising as the dipolar interactions in a pancake-shaped DBEC with $\alpha=0$ reduce to pure contact-like form [53. And, the DBEC is equivalent to the condensate interacting by pure contact interactions characterized by $s$-wave scattering length equal to $a+2 a_{\mathrm{dd}}$, where $a$ and $a_{\mathrm{dd}}$ are the $s$-wave scattering and dipolar lengths of the DBEC. Now, the frequency of rotation of the vortex for $\alpha=\pi / 4$ is greater than for $\alpha=0$. This is due to the fact that in a homogeneous superfluid bounded by a circular boundary, the frequency of rotation of the point vortex is [54]

$$
f=\frac{1}{4 \pi^{2}\left(R^{2}-r_{0}^{2}\right)},
$$

where $R$ is the radius of the circular region and $r_{0}$ is the position of the vortex. Approximating the radius of the circular region by the root mean square radius of the condensate $\left(R=\sqrt{\int r^{2}|\psi(\mathbf{r})|^{2} d x d y}\right)$, the ratio of rotation frequencies of the vortices initially located at $(1.55,0)$ for $\alpha=\pi / 4$ to $\alpha=0$ is 1.18 and is in very good agreement with the value 1.19 obtained from the first cycle in the bottom panel of Fig. 1. As the vortex moves away from the center of the trap, the frequency increases due to the decrease in the denominator of Eq. (7), and this is consistent with the dynamics shown in bottom panel of Fig. 1. where the adjacent peaks start coming closer to each other with the progress of time. For the same initial position of the vortex, the rate of decay of the vortex is more for $\alpha=\pi / 4$ as is shown in Fig. 2(a), where the radial coordinate of the vortex is plotted as a function of time.

In order to calculate the the decay rate, we fit $r=$ $r_{0} \exp \left(\gamma_{d} t\right)$ over the numerically calculated radial coordinate of the vortex, where $\gamma_{d}$ is decay rate of the vortex initially located at $r_{0}$. For the vortex initially located at $r_{0}=1.55 a_{\text {osc }}$, the decay rates obtained from this fitting are $1.263 \times 10^{-3}$ and $1.098 \times 10^{-3}$ for $\alpha=\pi / 4$ and $\alpha=0$ respectively. For the vortex initially located at $r_{0}=1.0 a_{\mathrm{osc}}$, the decay rates are slightly lower, $\gamma_{d}=1.261 \times 10^{-3}$ for $\alpha=\pi / 4$ and $\gamma_{d}=1.094 \times 10^{-3}$ for $\alpha=0$. Hence the decay rate is suppressed, although 

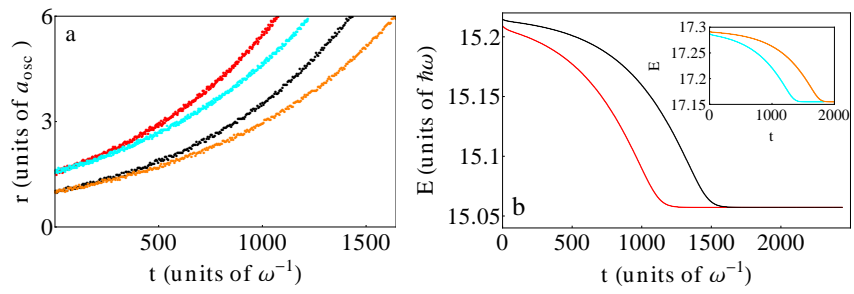

FIG. 2: The left figure shows the dynamics of single vortex in a DBEC of ${ }^{52} \mathrm{Cr}$ with $a=50 a_{0}, a_{d d}=16 a_{0}$, whereas the figure on right shows the corresponding variation in the energy of the condensate. The angle $\alpha=0$ for the cyan and orange curves, whereas $\alpha=\pi / 4$ for red and black curves. The upper and lower sets of curves correspond to two different initial locations of the vortices.

marginally, as the vortex moves nearer to the center of the trap. The suppression of the decay rate as the vortex moves closer to the trap center is also observed in condensates interacting via contact interactions $[18,20]$. In the absence of dissipation at $T=0 \mathrm{~K}$, an off-center vortex traverses along an isoenergetic circular trajectory around the trap center due to the excitation of the anomalous or fundamental Kelvin mode [55]57. The non-zero dissipation at finite temperatures slowly reduces the energy of the condensate, which is shown in Fig. 2(b), by making the vortex execute a spiral trajectory towards the edge of the condensate [58, 59. This leads to a vortex-free state which now is energetically stable.

Dynamics of a corotating vortex pair: In order to study the dynamics of two corotating vortices, we first generate the minimum energy solution by solving Eq. (5) in imaginary time $\tau$ in the absence of any dissipation. As in the case of a single vortex, we transform $\psi$ after each iteration in time as

$$
\begin{aligned}
\psi(\tau+\delta \tau)= & \exp \left\{i \left[\tan ^{-1}\left(\frac{y-y_{1}}{x-x_{1}}\right)\right.\right. \\
& \left.\left.+\tan ^{-1}\left(\frac{y-y_{2}}{x-x_{2}}\right)\right]\right\}|\psi(\tau)|,
\end{aligned}
$$

here $\left(x_{1}, y_{1}\right)$ and $\left(x_{2}, y_{2}\right)$ are the locations of the two vortices. The solution obtained by this method for the two vortices initially located at $\left(-1.0 a_{\mathrm{osc}},-1.88 a_{\mathrm{osc}}\right)$ and $\left(1.0 a_{\text {osc }}, 1.88 a_{\text {osc }}\right)$ is shown in Fig. 3

This minimum energy solution is now evolved in real time in the presence of dissipation. In the case of a corotating vortex pair, the dynamics depends upon the initial asymmetry in the location of the vortices. For the two vortices symmetrically located about origin, i.e. $x_{2}=-x_{1}$ and $y_{2}=-y_{1}$ as is the case for the solution shown in Fig. 3 . both the vortices decay at the same rate and slowly spiral out of the condensate like a single vortex as is shown by red dots in Fig. 4(a); the full dynamics is shown in Fig. $5(a)$ and (b). The corresponding variation in the energy of the system is shown by the red curve in Fig. 4(b).
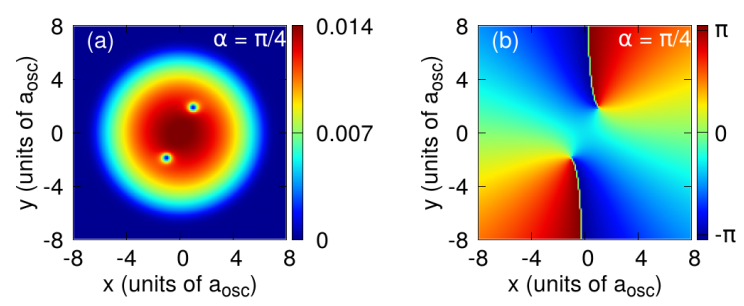

FIG. 3: (a) shows the density and (b) shows the phase of the DBEC of ${ }^{52} \mathrm{Cr}$ atoms with $a=50 a_{0}$ and $a_{\mathrm{dd}}=16 a_{0}$. The angle $\alpha=\pi / 4$.

In this case, we can define the decay rate as in the case of a single vortex by fitting $r=r_{0} \exp \left(\gamma_{d} t\right)$ over the numerical values of radial coordinates. The decay rate thus obtained is $1.35 \times 10^{-3}$.
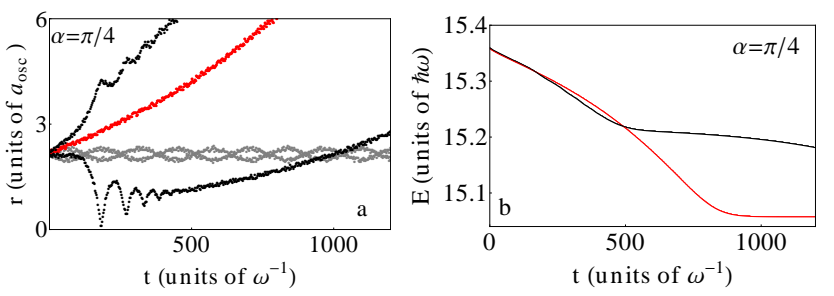

FIG. 4: The left figure shows the radial coordinates of the two corotating vortices as a function of time in a DBEC of ${ }^{52} \mathrm{Cr}$ with $a=50 a_{0}, a_{d d}=16 a_{0}$. The right figure shows the corresponding variation in the energy of the condensate with time. Red dots and line correspond to the vortices which are symmetrically located about origin at equal radial distances, whereas for the black dots and line there is a slight asymmetry in the locations of the two vortices. The value of dissipation $\gamma=0.0023$, except for the gray dots in the left figure, which show the radial coordinates of the asymmetrically located vortices as a function of time in the absence of dissipation.

In case the vortices are not symmetrically located about origin, the dynamics becomes qualitatively different. Here the two vortices don't decay at the same rate. Instead of this, the decay rate of one of the vortex is suppressed which starts moving closer to the center of the trap, whereas the decay rate of the second vortex is increased as is shown by black dots in Fig. 4(a). The initial coordinates of the vortices in this case are $\left(-1.0 a_{\mathrm{osc}},-1.88 a_{\mathrm{osc}}\right)$ and $\left(1.1 a_{\mathrm{osc}}, 1.77 a_{\mathrm{osc}}\right)$. If one examines the variation in energy in this case, shown by the black curve in Fig. 4(b), there are two distinct regions. For the region $t \lesssim 490 \omega^{-1}$, energy of the asymmetric vortex pair decays faster than the symmetric vortex pair. This is due to the increase in the separation between the 

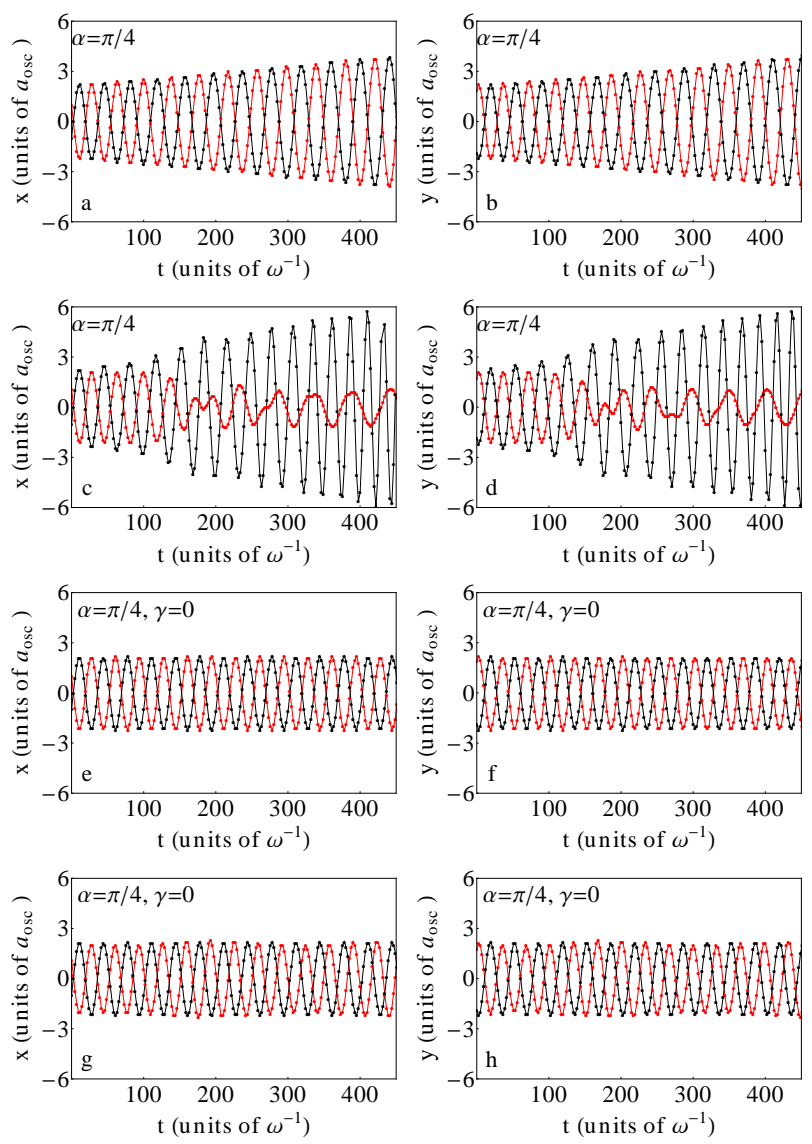

FIG. 5: The first row shows the dynamics of the two corotating vortices initially located at $\left(-1.0 a_{\text {osc }},-1.88 a_{\text {osc }}\right)$ and $\left(1.0 a_{\text {osc }}, 1.88 a_{\text {osc }}\right)$ at finite $T$, whereas third row shows the dynamics of the pair at $T=0 \mathrm{~K}$. The second row shows the dynamics of the two corotating vortices initially located at $\left(-1.0 a_{\mathrm{osc}},-1.88 a_{\mathrm{osc}}\right)$ and $\left(1.1 a_{\mathrm{osc}}, 1.77 a_{\mathrm{osc}}\right)$ at finite $T$, whereas the fourth row shows the dynamics of the pair at $T=0 \mathrm{~K}$.

two vortices leading to the decrease in the inter-vortex interaction energy. The faster decaying vortex ultimately exits out of the condensate leaving a single vortex, which now decays as a single vortex after $t \gtrsim 490 \omega^{-1}$. The full dynamics for the symmetrically and asymmetrically located pair of vortices is shown in Figs. 5(a-d). The finite temperature dynamics is in significant contrast to the dynamics at $T=0$ Kelvin $(\gamma=0)$, which is shown in Figs. [5(e-h). The radial coordinates of the asymmetrically located vortices at $T=0$ as a function of time are shown by grey dots in Fig. 4(a). It is evident that there are oscillations in the radial coordinates of the asymmetrically located vortices at $T=0 \mathrm{~K}$. From the Figs. 4(a) [the dynamics depicted by black dots], 5(c), and 5(d), it is evident that from the very beginning, one of the vortex starts moving towards the center, while the other starts moving towards the edge of the condensate. This, however, is not the case always. We find that for a different set of initial vortex locations, the component vortices may alternatively end up nearer to the trap center, espe- cially during the initial stages of the dynamics. This is evident from the black dots in Fig. 6, where the dynamics of two vortices initially located at $\left(-1.55 a_{\mathrm{osc}}, 0.0\right)$, $\left(1.0 a_{\text {osc }}, 0.0\right)$ is shown. Even when the initial asymmetry is decreased, there are radial oscillations (see red dots in Fig. 6). These radial oscillations are reminiscent of the dynamics of asymmetrically located vortices at zero temperature, see the dynamics depicted by gray dots in Fig. 4(a), 23. Due to this one of the vortices ends up decaying faster than the other. At finite temperature, the random fluctuations will invariably lead to the asymmetry in locations of the component vortices [23].

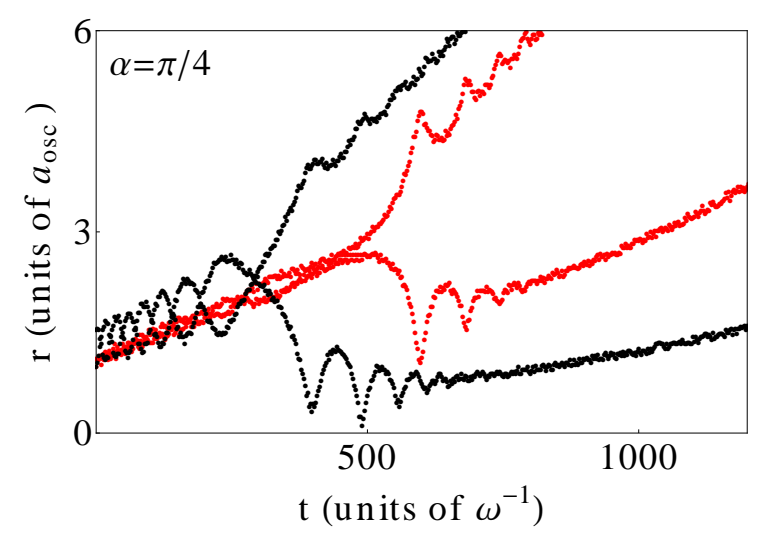

FIG. 6: Red dots are the radial coordinates of the corotating vortices initially located at $(-1.1,0.0),(1.0,0.0)$. The black dots are the radial coordinates of the corotating vortices initially located at $(-1.55,0.0),(1.0,0.0)$. The period during which the two black (red) dotted curves cross each other is qualitatively similar to the zero temperature dynamics as shown by the gray dots in Fig. 4(a).

Effect of anisotropic vortex-vortex interactions on the $d y$ namics: It was pointed out in Ref. 60] that if the magnetic dipoles have non-zero component on the plane of the condensate, the interactions between the vortices become anisotropic. In order to study the effect of anisotropic interactions on the dynamics of corotating vortices, we consider the dynamics of the vortex pair for three different initial locations. These are (a) $\left(-\sqrt{1.1^{2}+2.21^{2}}, 0\right)$, $\left(\sqrt{0.5^{2}+1.1^{2}}, 0\right)$; (b) $(-1.1,-2.21),(0.55,1.1)$; and (c) $\left(0,-\sqrt{1.1^{2}+2.21^{2}}\right),\left(0, \sqrt{0.5^{2}+1.1^{2}}\right)$ in scaled units. In these three cases, the components vortices have the same radial coordinates, and the relative position vector $\mathbf{r}_{2}-\mathbf{r}_{1}$ makes an angle $0, \pi / 4$ or $\pi / 2$ with the $x$-axis. Let us denote this angle by $\phi$. If $\alpha=\pi / 4$, the dynamics for these three cases is different as is shown in Fig.77(a). This is due to different interaction energy between the two vortices for these three cases. The interaction energy between the two vortices is defined as 60 .

$$
E_{\text {int }}\left(\mathbf{r}_{2}-\mathbf{r}_{1}\right)=E_{2}\left(\mathbf{r}_{1}, \mathbf{r}_{2}\right)-E_{1}\left(\mathbf{r}_{1}\right)-E_{1}\left(\mathbf{r}_{2}\right),
$$


where $E_{j}$ with $j=1,2$ is the energy associated with $j$ vortices in the system and is equal to total energy of the system minus the energy of the vortex free state. The interaction energy thus calculated are $0.0232 \hbar \omega$ for (a), $0.0229 \hbar \omega$ for (b), and $0.0228 \hbar \omega$ for (c). We checked the precision of these results upto four decimal places using two different spatial and imaginary time step sizes to solve the DGPE in imaginary time - (a) $\delta x=\delta y=0.11 a_{\text {osc }}$ and $\delta \tau=0.0006$ and (b) $\delta x=\delta y=0.055 a_{\text {osc }}$ and $\delta \tau=0.0002$. Hence the interaction energy is maximum for the vortices located along $x$-axis and minimum when they are located along $y$-axis in agreement with Ref. 60]. This anisotropy in the vortex interaction leads to different dynamics in the aforementioned three cases.
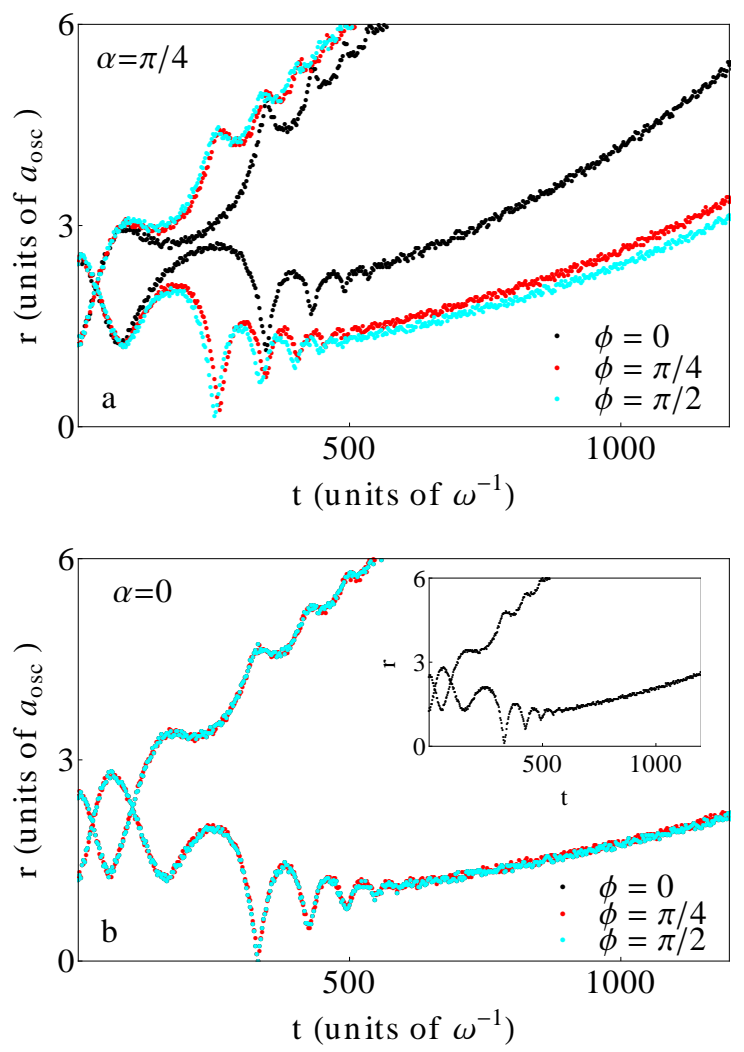

FIG. 7: The dynamics of a corotating vortex pair in a DBEC of $1.5 \times 10^{5}{ }^{52} \mathrm{Cr}$ atoms with $a=50 a_{0}, a_{d d}=16 a_{0}$ for three different initial orientations. The angle $\alpha=\pi / 4$ for (a) and $\alpha=0$ for (b). The angle $\phi$ is equal to $0, \pi / 4$, and $\pi / 2$ for black, red, and cyan dots respectively. The inset in (b) shows the dynamics of the two corotating vortices which are located diametrically opposite to each other at radial coordinates $r_{1}=\sqrt{1.1^{2}+2.21^{2}}, r_{2}=\sqrt{0.5^{2}+1.1^{2}}$ and arbitrary $\phi$ in a condensate of ${ }^{52} \mathrm{Cr}$ atoms with $a=82 a_{0}$ and $a_{d d}=0$.
In case $\alpha=0$, the vortex-vortex interaction is isotropic and is manifested by same dynamics for the aforementioned three cases as is shown in Fig. 7(b). The initial vortex-vortex interaction energy in this case is $0.0241 \hbar \omega$ irrespective of the value of $\phi$. As mentioned earlier, a pancake-shaped DBEC with $\alpha=0$ is equivalent to a condensate interacting via pure contact interactions. In order to compare the dynamics of the corotating vortex pair in such a condensate with that of the DBEC, we consider a condensate consisting of $1.5 \times 10^{5}$ atoms of ${ }^{52} \mathrm{Cr}$ with $a=82 a_{0}$ and $a_{\mathrm{dd}}=0$. The dynamics of two corotating vortices, initially located diametrically opposite to each other at radial coordinates $r_{1}=\sqrt{1.1^{2}+2.21^{2}}$, $r_{2}=\sqrt{0.5^{2}+1.1^{2}}$ and any arbitrary $\phi$ is shown in the inset of Fig. 77(b). It is evident that the dynamics in two cases is same. This equivalence in dynamics of vortices in the pancake-shaped DBEC with $\alpha=0$ and the condensate interacting with pure contact interactions does not depend upon $\gamma$, and hence is true even at $T=0 \mathrm{~K}$ $(\gamma=0)$.

\section{SUMMARY OF RESULTS}

We have studied the dynamics of a single vortex and two corotating vortices in the quasi two-dimensional dipolar condensates in the presence of dissipation. A single vortex in the presence of dissipation spirals out of the condensate due to the energetic instability. We observe that making the dipolar interactions partially attractive by changing the polarization direction, leads to the decrease in size of the condensate and faster decay rate for a single vortex. In the case of two symmetrically located corotating vortices, the finite temperature dynamics is qualitatively similar to the single vortex, and the decay rate of the each vortex is equal. On the other hand, the introduction of the slight asymmetry in the initial locations leads to the different dynamics. In this case, the decay rate of the one of the vortex is suppressed at the cost of the other. For the dipoles not oriented normal to the condensate plane, the vortex-vortex interactions are no longer isotropic. We observe that even a small difference in vortex-vortex interaction energy can lead to the perceptible difference in the dynamics at finite temperature.

\section{Acknowledgments}

We thank Prof. S. K. Adhikari for useful comments and suggestions. This work is financed by Fundação de Amparo à Pesquisa do Estado de São Paulo (FAPESP) under Contract No. 2013/07213-0. 
[2] K. W. Madison, F. Chevy, W. Wohllenben, and J. Dalibard, Phys. Rev. Lett. 84, 806 (2000); K. W. Madison, F. Chevy, V. Bretin, and J. Dalibard, Phys. Rev. Lett. 86, 4443 (2001).

[3] C. Raman, J. R. Abo-Shaeer, J. M. Vogels, K. Xu, and W. Ketterle, Phys. Rev. Lett. 87, 210402 (2001).

[4] J. R. Abo-Shaeer, C. Raman, J. M. Vogels, and W. Ketterle, Science 292, 476 (2001).

[5] A. E. Leanhardt, A. Görlitz, A. P. Chikkatur, D. Kielpinski, Y. Shin, D. E. Pritchard, and W. Ketterle, Phys. Rev. Lett. 89, 190403 (2002).

[6] T. W. Neely, E. C. Samson, A. S. Bradley, M. J. Davis, and B. P. Anderson, Phys. Rev. Lett. 104, 160401 (2010).

[7] B. P. Anderson, P. .C. Haljan, C. A. Regal, D. L. Feder, L. A. Collins, C. W. Clark, and E. A. Cornell, Phys. Rev. Lett. 86, 2926 (2001).

[8] C. Weiler, T. W. Neely, D. R. Scherer, A. .S. Bradley, M. J. Davis, and B. P. Anderson, Nature 455, 948 (2008).

[9] D. V. Freilich, D. M. Bianchi, A. M. Kaufman, T. K. Langin, D. S. Hall, Science 329, 1182 (2010).

[10] R. Navarro, R. Carretero-González, P. J. Torres, P. G. Kevrekidis, D. J. Frantzeskakis, M. W. Ray, E. Altuntas, and D. S. Hall, Phys. Rev. Lett. 110, 225301 (2013).

[11] S. Middelkamp, P. J. Torres, P. G. Kevrekidis, D. J. Frantzeskakis, R. Carretero-González, P. Schmelcher, D. V. Freilich, and D. S. Hall, Phys. Rev. A 84, 011605(R) (2011).

[12] P. J. Torres, P. G. Kevrekidis, D. J. Frantzeskakis, R. Carretero-González, P. Schmelcher, D. S. Hall, Phys. Lett. A 375, 3044 (2011).

[13] P. Kuopanportti, Jukka A. M. Huhtamäki, and M. Möttönen, Phys. Rev. A 83, 011603(R) (2011).

[14] S. Gautam, P. Muruganandam, and D. Angom, J. Phys. B 45, 055303 (2012); S. Gautam, P. Muruganandam, and D. Angom, Phys. Lett. A 377, 378 (2013).

[15] H. Schmidt, K. Góral, F. Floegel, M. Gajda, and K. Rzazewski, J. Opt. B: Quantum Semiclass. Opt. 5 S96, (2003).

[16] E. J. Madarassy and C. F. Barenghi, J. Low Temp. Phys. 152, 122 (2008).

[17] B. Jackson, N. P. Proukakis, C. F. Barenghi, and E. Zaremba, Phys. Rev. A 79, 053615 (2009).

[18] A. J. Allen, E. Zaremba, C. F. Barenghi, and N. P. Proukakis, Phys. Rev A 87, 013630 (2013).

[19] T. M. Wright, A. .S. Bradley, and R. J. Ballagh, Phys. Rev. A 80, 053624 (2009).

[20] S. J. Rooney, A. S. Bradley, and P. B. Blakie, Phys. Rev. A 81, 023630 (2010).

[21] R. .A. Duine, B. W. A. Leurs, and H. T. C. Stoof, Phys. Rev. A 69, 053623 (2004).

[22] D. Yan, R. Carretero-González, D. J. Frantzeskakis, P. G. Kevrekidis, N. P. Proukakis, D. Spirn, arXiv:1309.7943.

[23] S. Gautam, Arko Roy, and Subroto Mukerjee, Phys. Rev. A 89, 013612 (2014).

[24] A. Griesmaier, J. Werner, S. Hensler, J. Stuhler, and T. Pfau, Phys. Rev. Lett. 94, 160401 (2005); A. Griesmaier, J. Stuhler, and T. Pfau, Applied Physics B 82, 211 (2006).

[25] T. Lahaye, T. Koch, B. Fröhlich, M. Fattori, J. Metz, A. Griesmaier, S. Giovanazzi, and T. Pfau, Nature 448, 672 (2007).

[26] T. Koch, T. Lahaye, J. Metz, B. Fröhlich, A. Griesmaier, and T. Pfau, Nature Physics 4, 218 (2008).

[27] M. Lu, N. Q. Burdick, S. H. Youn, and B. L. Lev, Phys. Rev. Lett. 107, 190401 (2011).

[28] K. Aikawa, A. Frisch, M. Mark, S. Baier, A. Rietzler, R. Grimm, and F. Ferlaino, Phys. Rev. Lett. 108, 210401 (2012).

[29] T. Lahaye, C. Menotti, L. Santos, M. Lewenstein, and T. Pfau, Rep. Prog. Phys. 72, 126401 (2009).

[30] M. A. Baranov, M. Dalmonte, G. Pupillo, and P. Zoller, Chem. Rev. 112, 5012 (2012).

[31] L. Santos, G. V. Shlyapnikov, M. Lewenstein, Phys. Rev. Lett. 90, 250403 (2003).

[32] R. M. Wilson, S. Ronen, J. L. Bohn, and H. Pu, Phys. Rev. Lett. 100, 245302 (2008).

[33] R. M. Wilson, S. Ronen, and J. L. Bohn, Phys. Rev. Lett. 104, 094501 (2010).

[34] U. R. Fischer, Phys. Rev. A 73, 031602(R) (2006).

[35] L. Landau, J. Phys. (Moscow) 5, 71 (1941).

[36] S. Yi and H. Pu, Phys. Rev. A 73, 061602(R) (2006).

[37] S. Ronen, D. C. E. Bortolotti, and J. L. Bohn, Phys. Rev. Lett. 98, 030406 (2007).

[38] R M. Wilson, S. Ronen, and J. L. Bohn, Phys. Rev. A 80, 023614 (2009).

[39] A. Boudjemâa and G. V. Shlyapnikov, Phys. Rev. A 87, 025601 (2013)

[40] C. Ticknor, R. M. Wilson, and J. L. Bohn, Phys. Rev. Lett. 106, 065301 (2011).

[41] P. Muruganandam and S. K. Adhikari, Phys. Lett. A 376, 480 (2012).

[42] S. Gautam and Subroto Mukerjee, arXiv:1401.0268

[43] M. Abad, M. Guilleumas, R. Mayol, M. Piand D. M. Jezek, Phys. Rev. A 79, 063622 (2009); M. Abad, M. Guilleumas, R. Mayol, M. Piand D. M. Jezek, Phys. Rev. A 81, 043619 (2010).

[44] R. M. W. van Bijnen, D. H. J. O'Dell, N. G. Parker, and A. M. Martin, Phys. Rev. Lett. 98, 150401 (2007); R. M. W. van Bijnen, A. J. Dow, D. H. J. O'Dell, N. G. Parker, and A. M. Martin, Phys. Rev. A 80, 033617 (2009).

[45] F. Malet, T. Kristensen, S. M. Reimann, and G. M. Kavoulakis, Phys. Rev. A 83, 033628 (2011).

[46] R. Kishor Kumar and P. Muruganandam, J. Phys. B 45, 215301 (2012).

[47] L. P. Pitaevskii, Zh. Eksp. Teor. Fiz. 35, 408 (1958) [Sov. Phys. JETP 35, 282 (1959)].

[48] A. A. Penckwitt, R. J. Ballagh, and C. W. Gardiner, Phys. Rev. Lett. 89, 260402 (2002).

[49] P. B. Blakie, A. S. Bradley, M. J. Davis, R. J. Ballagh, and C. W. Gardiner, Adv. Phys. 57, 363 (2008).

[50] P. Pedri and L. Santos, Phys. Rev. Lett. 95, 200404 (2005).

[51] P. Muruganandam and S. K. Adhikari, Laser Physics 22, 813 (2012).

[52] W. Bao, D. Jaksch, and P. A. Markowich, J. Comp. Phys. 187, 318 (2003).

[53] N. G. Parker and D. H. J. O'Dell, Phys. Rev. A 78, 041601(R), 2008.

[54] S. V. Alekseenko, P. A. Kuibin, and V. L. Okulov, Theory of Concentrated Vortices Springer (2007).

[55] R. J. Dodd, K. Burnett, M. Edwards, and C. W. Clark, Phys. Rev. A 56, 587 (1997).

[56] T. P. Simula and K. Machida, Phys. Rev. A 82, 063627 (2010); T. P. Simula, ibid. 87, 023630 (2013).

[57] S. Middelkamp, P. G. Kevrekidis, D. J. Frantzeskakis, 
R. Carretero-González, P. Schmelcher, J. Phys. B 43, 155303 (2010).

[58] D. S. Rokhsar, Phys. Rev. Lett. 79, 2164 (1997).

[59] A. A. Svidzinsky and A. L. Fetter, Phys. Rev. Lett. 84, 5919 (2000).
[60] B. C. Mulkerin, R. M. W. van Bijnen, D. H. J. O'Dell, A. M. Martin, and N. G. Parker, Phys. Rev. Lett. 111, 170402 (2013). 